typeset using JPSJ.sty $<$ ver.1.0b $>$

\title{
Spin Splitting in de Haas-van Alphen Oscillation in Two-Dimensional Two-Band Systems
}

\author{
Keita Kishigi, Yasumasa Hasegawa and Mitake MiYazaKi \\ Faculty of Science, Himeji Institute of Technology, Akou-gun, Hyogo 678-1297, Japan
}

(Received January 6, 2018)

\begin{abstract}
We study the effects of the Zeeman term on the de Haas van Alphen (dHvA) oscillation in two-dimensional two-band systems. We found that the Fourier transform amplitudes of the oscillations are not described by the spin reduction factor in the Lifshitz-Kosevich formalism in two-dimensional systems. The anomalous dependence on the effective $g$-factor can be observed by tilting-angle dependence of the $\mathrm{dHvA}$ oscillation in quasi-two-dimensional organic conductors and $\mathrm{Sr}_{2} \mathrm{RuO}_{4}$.
\end{abstract}

KEYWORDS: dHvA Oscillation, $\mathrm{Sr}_{2} \mathrm{RuO}_{4}$, Quasi-Two-Dimensional Organic Conductors, Zeeman effect, Magnetic Breakdown

The magnetization and magnetoresistance oscillate as a function of the inverse of the magnetic field $(H)$ with the period proportional to the extreme area $(f)$ of the closed orbit on the Fermi surface. These phenomena are known as the Shubnikov-de Haas ( $\mathrm{SdH})$ oscillation and the de Haas-van Alphen (dHvA) oscillation. The dHvA oscillation can be described in the semiflassical theory as Lifshitz and Kosevich (LK) formula. 1. 2 )

In this study, we consider the system at the clean limit and zero temperature. In the two-dimensional singleband system, the magnetization, $M^{s}(\mu, H)$, for the fixed chemical potential $(\mu)$ is given by

$$
\begin{aligned}
M^{s}(\mu, H) & =-\sum_{p=1}^{\infty} R_{p}^{\mu} \frac{1}{p} \sin p\left(\frac{f}{H}-\pi\right), \\
R_{p}^{\mu} & =\cos \left(\frac{\pi}{2} p g \frac{m}{m_{0}}\right),
\end{aligned}
$$

where $R_{p}^{\mu}$ is the reduction factor due to electron spin, $g \simeq 2$ is the $g$-factor, $m$ is the cyclotron effective mass and $m_{0}$ is the free electron mass. The spin factor is caused by the phase shift in the oscillation for up and down spins. When the Zeeman splitting becomes a half of the Landau level spacing, the amplitude of the oscillation of the fundamental frequency $(A=1)$ becomes zero. Eq. (1) is called the LK formula H $^{2}$ The experiments are performed in the multi energy-band system with the fixed electron number $(N)$, where the chemical potential varies as a function of the magnetic field. However, the oscillation of the chemical potential is very small when the Fermi surface has a three-dimensional shape. Then we can apply eq. (1) or the superposition of eq. (1) with some frequencies even for the system with fixed electron number.

On the other hand, if the system is two-dimensional or the interlayer coupling is much smaller than the Landau level spacings (this condition is satisfied in the quasitwo-dimensional system in the strong magnetic field), the oscillation of the chemical potential plays important roll. The magnetization for the fixed electron number is given by国

$$
M^{s}(N, H)=\sum_{p=1}^{\infty} R_{p}^{N} \frac{1}{p} \sin p \frac{f}{H},
$$

in the two-dimensional single-band system. The spin reduction factor in this case is s $^{4}$

$\left|R_{p}^{N}\right|^{2}=\left\{\begin{array}{cc}\left|1-2\left(g m / 2 m_{0}-\left[g m / 2 m_{0}\right]\right)\right|^{2} & \text { for odd } p_{(4)} \\ 1 & \text { for even } p,\end{array}\right.$

where $\left[\mathrm{gm} / 2 m_{0}\right]$ is an integer part of $g m / 2 m_{0}$. The spin reduction factor, $R_{p}^{N}$, is the periodic function of $\mathrm{gm} / 2 \mathrm{~m}_{0}$ but is not the cosine function.

If the magnetic field is tilted by $\theta$ from the z-axis, the spin reduction factor is given by replacing $g$ with $g / \cos \theta$ in eqs. (2) and (4). The reduction factor for the $p$ th harmonics, $R_{p}^{\mu}$, is zero when $\cos \theta=\left(\mathrm{pgm} / \mathrm{m}_{0}\right) /(2 n+$ 1) with integer $n$. When $\cos \theta=\left(g m / m_{0}\right) /(2 n+1)$, $R_{p}^{N}$ for odd $p$ is zero. The tilting angle, $\theta$, when $R_{p}^{\mu}$ or $R_{p}^{N}$ becomes zero is called spin splitting zero condition 2 ) which can be measured by tilting the magnetic field.

When two energy bands exist in the two-dimensional system, the magnetization is not described by the superposition of the magnetization for the single bandeg.

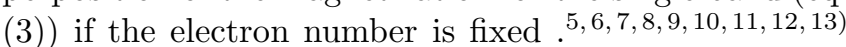
One of the authors 目目 Harrisonet al. 18 Sandu et al. (9) Han et al.10 and Fortin et al11) treat the magnetic breakdown model as shown in Fig. 1(a), where the $\beta$ orbit is obtained by the tunneling through the zone gap. Nakano ${ }^{12}$ ) and Alexandrov and Bratkovsky ${ }^{3}$ ) have calculated the dHvA oscillation by using the independent two-band model, in which there exist two closed orbits ( $\alpha$ and $\beta$ orbits) shown in Fig. 1 (b). From these

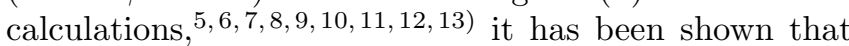
there exist frequencies in the magnetization $(M(N, H))$, which are forbidden in the semiclassical theory. One of these forbidden frequencies corresponds to the area of the $\beta-\alpha$ orbit. On the other hand, the forbiddenoscillations

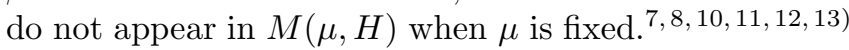
These forbidden oscillations are caused by the chemical 
potential oscillation.

The existence of the $\beta-\alpha$ oscillation in the magnetization has been observed experimentally in quasitwo-dimensional materials with two or three energy bands, 14 -(BEDT-TTF $)_{2} \mathrm{Cu}\left(\mathrm{NCS}_{2}, 15,16\right) \quad \alpha$-(BEDT$\mathrm{TTF})_{2} \mathrm{KHg}(\mathrm{SCN})_{4}{ }^{17}$ ) and $\mathrm{Sr}_{2} \mathrm{RuO}_{4}$ (18)

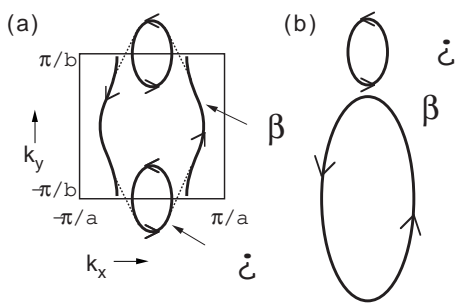

Fig. 1. (a) Fermi surface in the magnetic breakdown system. (b) Fermi surface in the two-band system.

The spin was neglected to simplify the calculation 5. $6,0,8,6,10,11,12,13)$ In order to study how the spin affects the "forbidden" $\beta-\alpha$ oscillation and quantum magnetic oscillations $(\alpha, \beta, \beta+\alpha, 2 \alpha$ oscillations, etc.) in the two-dimensional magnetic breakdown systems, we have studied $M\left(N_{\sigma} H\right)$ in the tight-binding model quantummechanically.3) This model can be applied to the quasitwo-dimensional organic conductors. We found that the Fourier transform amplitudes (FTAs) of the $\beta+\alpha$ oscillation is enhanced due to the Zeeman effect, which is quite different from the spin reduction factors, eq. (2) or eq. (4). However, as far as we know, the effect of the Zeeman term in the two-dimensional two-band model has never been discussed systematically.

In this letter, we calculate $M(N, H)$ at $T=0$ for the two-dimensional two-band model, where the magnetic breakdown is neglected. In this case neither chemical potential nor electron number for each band and each spin are fixed. Our purpose is to make clear how the oscillation in $M(N, H)$ are modulated by an electron spin. by

We study the model, whose energy at $H=0$ is given

$$
E_{i}(\mathbf{k})=\frac{\hbar^{2} k_{x}^{2}}{2 m_{i}^{x}}+\frac{\hbar^{2} k_{y}^{2}}{2 m_{i}^{y}}+\epsilon_{i}^{b},
$$

where $\epsilon_{i}^{b}$ is the bottom energy of the energy band and $i=\alpha$ and $\beta$ are the band index. The density of states for spin $\rho_{i}$ and the cyclotron effective mass $m_{i}$ are

$$
\rho_{i}=C m_{i}, m_{i}=\sqrt{m_{i}^{x} m_{i}^{y}},
$$

where $C=S / 2 \pi \hbar^{2}$ and $S$ is the real space area of the two-dimensional system.

When the magnetic field $\left(H_{0}\right)$ is applied with the tilt-

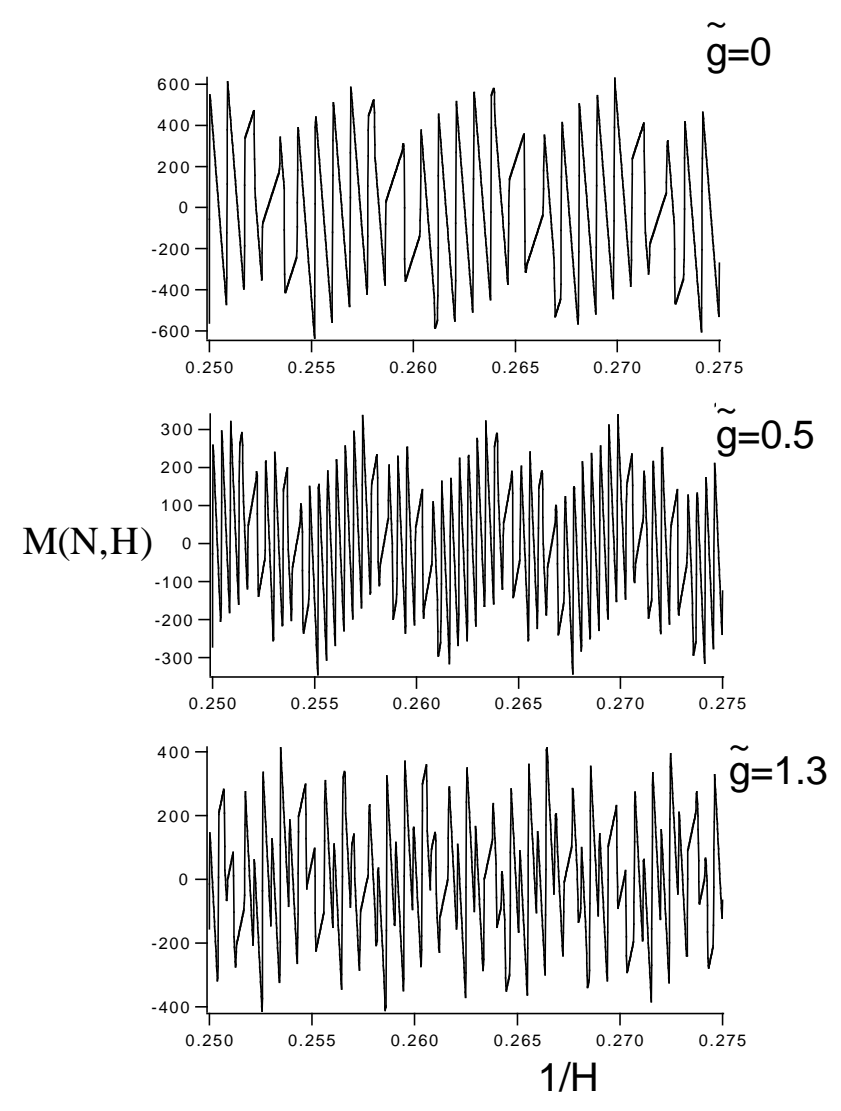

Fig. 2. $M(N, H)$ as a function of $1 / H$ when $\widetilde{g}=0,1.0$ and 2.6.

ing angle $\theta$, the $z$-component of the magnetic field is $H=H_{0} \cos \theta$. The energy spectrum is quantized as

$$
\epsilon_{i}(H, n, \sigma)=\hbar \omega_{i}\left(n+\frac{1}{2}+\sigma\left(\frac{m_{i}}{m_{0}}\right) \widetilde{g}_{i}\right)+\epsilon_{i}^{b},
$$

where $n$ is Landau index, $\omega_{i}=e H / m_{i} c, \sigma= \pm \frac{1}{2}$ and $\widetilde{g}_{i}=g_{i} / 2 \cos \theta$. We take $g_{\alpha}=g_{\beta}$ and $\widetilde{g}_{i}=\widetilde{g}$, although $g_{\alpha}$ and $g_{\beta}$ are not necessarily the same due to the spinorbit coupling. We set $e=\hbar=c=1$ and $C=1$.

When the electron number $(N)$ is fixed, we have to calculate the Helmholtz free energy $F(N, H)$, which is given by

$$
F(N, H)=\sum_{i} \rho_{i} \hbar \omega_{i} \sum_{n, \sigma} \epsilon_{i}(H, n, \sigma),
$$

where the summations are performed for the filled and partially filled energy levels. The electron number, $N$, is decided by the chemical potential, $\mu(0)$, at $H=0$. The magnetization is obtained by

$$
M(N, H)=-\partial F(N, H) / \partial H .
$$

We calculate $M(N, H)$ by changing the value of $\widetilde{g}$. When $\widetilde{g}=0, M(N, H)$ are reduced to the spinless model.12

We show $M(N, H)$ for various values of $\widetilde{g}$ in Fig. 2, where $m_{\alpha} / m_{0}=0.5, m_{\beta} / m_{0}=1.0, \epsilon_{\alpha}^{b}=680, \epsilon_{\beta}^{b}=0$ and $\mu(0)=1000$. We take $C=1$ and $0.25 \leq 1 / H \leq$ 0.275 , which corresponds to about 8 tesla and $\mu(0) \simeq 0.2$ 
$\mathrm{eV}$. In these parameters, the area of the $\alpha$ and $\beta$ orbits corresponds to $f_{\alpha}=160$ and $f_{\beta}=1000$.

We calculate the FTAs of $M(N, H)$, where there exit many combination frequencies, $\beta \pm \alpha, \beta \pm 2 \alpha, 2 \beta \pm \alpha$, $2 \beta \pm 2 \alpha$, in addition to $\alpha, \beta, 2 \alpha$ and $2 \beta$, which are shown in Fig. 3.
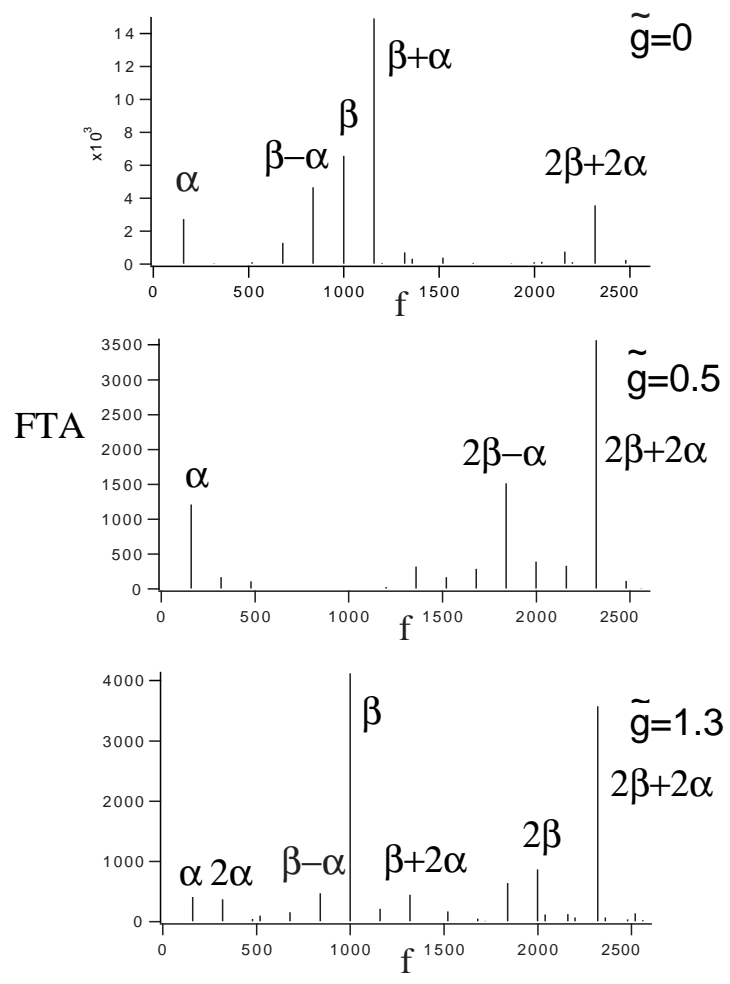

Fig. 3. The Fourier transform amplitudes of $M(N, H)$ in Fig. 2.

We show the FTAs of $M(N, H)$ as a function of $\widetilde{g}$ in Figs. 4 and 5. We also show FTAs for other parameters $\left(m_{\alpha} / m_{0}=0.65, m_{\beta} / m_{0}=1.0, \epsilon_{\alpha}^{b}=754, \epsilon_{\beta}^{b}=0\right.$ and $\mu(0)=1000)$ in Figs. 6 and 7.

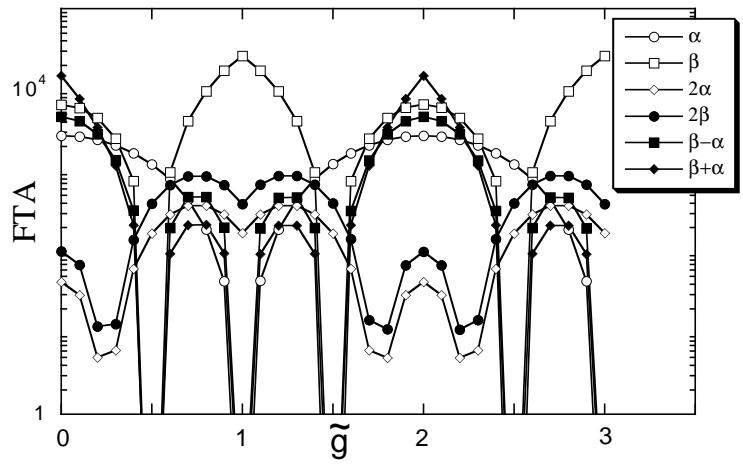

Fig. 4. The FTAs of some peaks $\left(f_{\alpha}, f_{2 \alpha}, f_{\beta}, f_{2 \beta}, f_{\beta-\alpha}\right.$ and $\left.f_{\beta+\alpha}\right)$ in $M(N, H)$ as a function of $\widetilde{g}$ for $m_{\alpha} / m_{0}=$ $0.5, m_{\beta} / m_{0}=1.0, \epsilon_{\alpha}^{b}=680$ and $\epsilon_{\beta}^{b}=0$.

It is clear from Fig. 4 that the $\widetilde{g}$-dependences of the FTAs are not described by the spin reduction factor for the single-band systems, eq. (4). For example, the FTAs

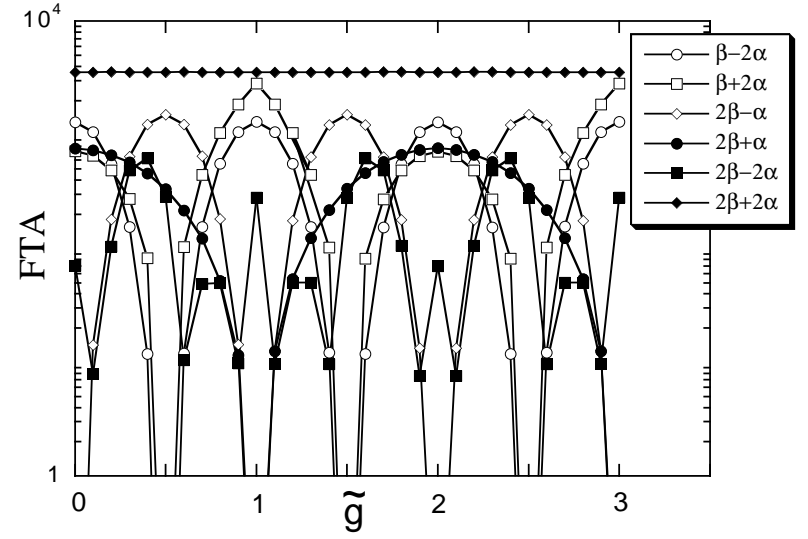

Fig. 5. The FTAs of some peaks $\left(f_{\beta-2 \alpha}, f_{\beta+2 \alpha}, f_{2 \beta-\alpha}, f_{2 \beta+\alpha}\right.$, $f_{2 \beta-2 \alpha}$ and $\left.f_{2 \beta+2 \alpha}\right)$ in $M(N, H)$ as a function of $g$ for $m_{\alpha} / m_{0}=$ $0.5, m_{\beta} / m_{0}=1.0, \epsilon_{\alpha}^{b}=680$ and $\epsilon_{\beta}^{b}=0$.

of the second harmonics oscillations ( $2 \alpha$ and $2 \beta$ oscillations) are not constant as a function of $\widetilde{g}$, which is not expected from eq. (4) for even $p$.

The FTAs of the fundamental oscillations $(\alpha$ and $\beta$ oscillations) become zero when $\widetilde{g}=\frac{1}{2}\left(m_{0} / m_{i}\right)(2 n+1)$, which can be seen in Figs. 4 and 6 . This is the same as the condition of the spin splitting zero given by eq. (4) for $p=1$.

We can see from Figs 4 and 6 that the $\beta+\alpha$ and $\beta$ $\alpha$ oscillations are suppressed when the FTAs of $\alpha$ or $\beta$ oscillations becomes zero. This is in contrast with the magnetic breakdown model (Fig. 4 in ref.B)). For example, in the magnetic breakdown model the FTA of the $\beta+\alpha$ oscillation is large at $\widetilde{g}=0.4$, when the $\beta$ oscillation is suppressed. The large amplitude of the $\beta+\alpha$ oscillation in the magnetic breakdown model might be understood as follows. The suppression of the $\beta$ oscillation is caused by the cancellation due to the $\pi$ phase difference between the oscillations for the up spin and the down spin in the semiclassical picture. This dose not result in the cancellation of the $\beta+\alpha$ oscillation in the magnetic breakdown systems because the $\beta+\alpha$ oscillation corresponds to the larger orbit caused by the tunneling and the phase difference between the up and down spins in the $\beta+\alpha$ orbit may not be $\pi$. In the two-band systems without the magnetic breakdown, the $\beta+\alpha$ oscillation is not caused by the larger orbit but the chemical potential oscillation. As a result, the $\beta+\alpha$ oscillation is suppressed in the two-band systems when $\alpha$ or $\beta$ oscillation is suppressed.

The FTA of the $2 \beta+2 \alpha$ oscillation is constant as a function of $\widetilde{g}$, which can be seen in Figs. 5 and 7 .

By tilting the magnetic field the suppression of the peak of the $\beta \pm \alpha$ oscillations and the constant peak of the $2 \beta+2 \alpha$ oscillation as a function of $\widetilde{g}$ may be observed in $\mathrm{Sr}_{2} \mathrm{RuO}_{4}$.

The Yamaji effect 19 ) should be also considered when the magnetic field is tilted if the Fermi surface has weak three-dimensionality. Nakand20) shows that the $\beta \pm \alpha$ oscillations become large due to the Yamaji effect when $\alpha$ and $\beta$ oscillations are enhanced in the spinless model. In quasi two-dimensional materials, the interplay between 
the spin effect studied in this paper and the Yamaji effect should be considered.

Ohmichi et al 21) have measured the FTAs of each oscillations in the magnetoresistance in $\mathrm{Sr}_{2} \mathrm{RuO}_{4}$, where there is no suppression of the peak of the $\beta \pm \alpha$ oscillations due to the spin although the enhancement of each oscillations due to Yamaji effect is seen. Our theory for the magnetization cannot be compared with their magnetoresistance experiment 21) because the Stark quantum interference oscillation 22,23) should be considered in the magnetoresistance. Yoshida et al.24 have measured the angle-dependence of the FTA of the $\alpha$ oscillation. We expect that the effect of the spin and quasi-twodimensionality can be observed in the angle-dependence of the FTAs of $\beta \pm \alpha$ and higher harmonic oscillations of the dHvA oscillation in $\mathrm{Sr}_{2} \mathrm{RuO}_{4}$.

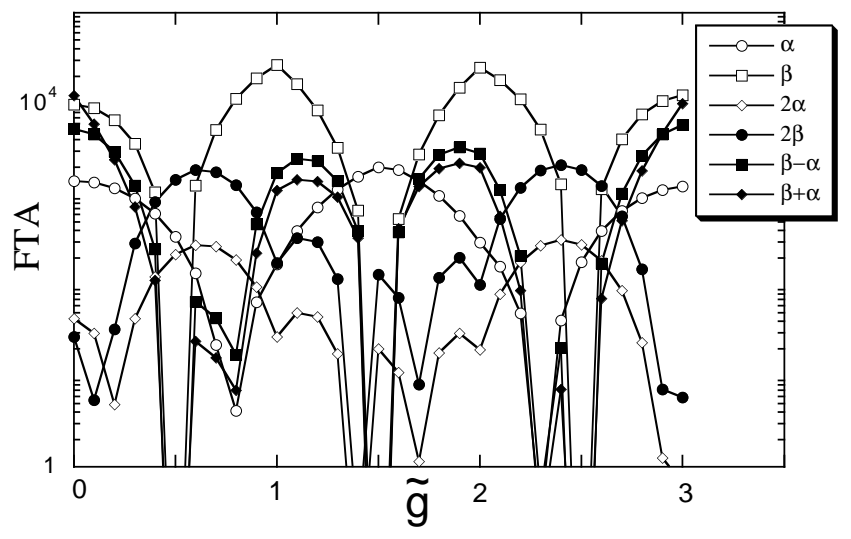

Fig. 6. The FTAs of some peaks $\left(f_{\alpha}, f_{2 \alpha}, f_{\beta}, f_{2 \beta}, f_{\beta-\alpha}\right.$ and $\left.f_{\beta+\alpha}\right)$ in $M(N, H)$ as a function of $\widetilde{g}$ for $m_{\alpha} / m_{0}=$ $0.65, m_{\beta} / m_{0}=1.0, \epsilon_{\alpha}^{b}=754$ and $\epsilon_{\beta}^{b}=0$.

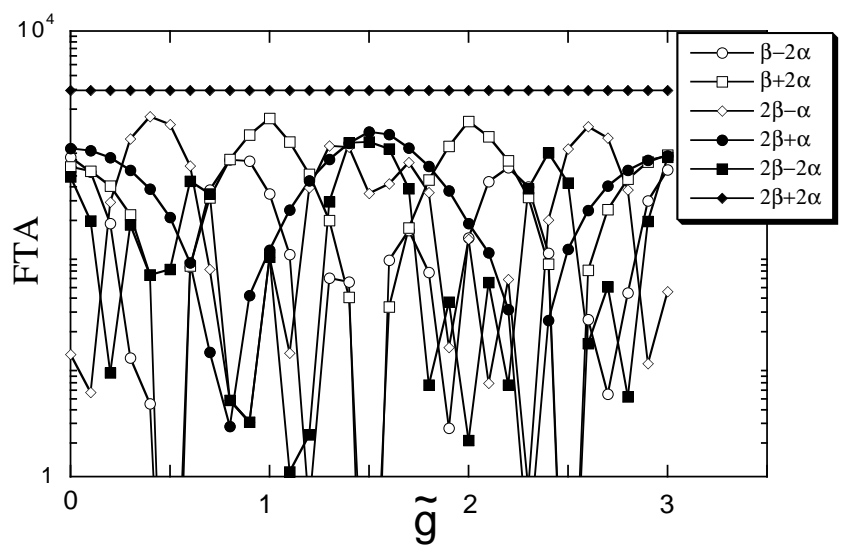

Fig. 7. The FTAs of some peaks $\left(f_{\beta-2 \alpha}, f_{\beta+2 \alpha}, f_{2 \beta-\alpha}, f_{2 \beta+\alpha}\right.$, $f_{2 \beta-2 \alpha}$ and $\left.f_{2 \beta+2 \alpha}\right)$ in $M(N, H)$ as a function of $g$ for $m_{\alpha} / m_{0}=$ $0.65, m_{\beta} / m_{0}=1.0, \epsilon_{\alpha}^{b}=754$ and $\epsilon_{\beta}^{b}=0$.

In conclusion, we study the dHvA oscillation including the Zeeman effect for the two-dimensional two-band model, where the magnetic breakdown is neglected. We find the anomalous $\widetilde{g}$-dependences of the FTAs of $\beta+\alpha$, $\beta-\alpha$ and $2 \beta+2 \alpha$ oscillations in $M(N, H)$, where $\widetilde{g}=$ $\left(g m_{i}\right) /\left(2 m_{0} \cos \theta\right)$. The $\beta \pm \alpha$ oscillations are suppressed when the $\alpha$ or $\beta$ oscillation disappears, and the FTA of the $2 \beta+2 \alpha$ oscillation is constant as a function of $\widetilde{g}$. The effect of the spin on $M(N, H)$ cannot be described by the spin reduction factor for the single-band (eq. (4)), whereas that in $M(\mu, H)$ is given by eq.(2). Even in the two-band model, however, the spin splitting zero condition for fundamental frequencies ( $\alpha$ and $\beta$ oscillations) in $M(N, H)$ is given by eq. (4). We expect that the $\widetilde{g}$ dependences of the amplitudes of these oscillations may be observed in the experiment of tilting magnetic field in two-dimensional multi-band system such as $\mathrm{Sr}_{2} \mathrm{RuO}_{4}$.

We would like to thank M. Nakano for valuable discussions. One of the authors (K. K.) was partially supported by Grant-in-Aid for JSPS Fellows from the Ministry of Education, Science, Sports and Culture. K. K. was financially supported by the Research Fellowships of the Japan Society for the Promotion of Science for Young Scientists.

[1] I. M. Lifshitz and A. M. Kosevich: Sov. Phys. JETP 2 (1956) 636.

[2] D. Shoenberg: Magnetic oscillation in metals (Cambridge University Press: Cambridge, 1984).

[3] K. Kishigi, Y. Hasegawa and M. Miyazaki: J. Phys. Soc. Jpn. 68 (1999) 1817.

[4] M. Nakano: preprint

[5] K. Machida, K. Kishigi and Y. Hori: Phys. Rev. B 51(1995) 8946.

[6] K. Kishigi, M. Nakano, K. Machida, and Y. Hori: J. Phys. Soc. Jpn. 64 (1995) 3043.

[7] K. Kishigi: J. Phys. Soc. Jpn. 66 (1997) 910.

[8] N. Harrison, J. Caulfield, J. Singleton, P. H. P. Reinders, F. Herlach, W. Hayes, M. Kurmoo and P. Day: J. Phys.: Condend. Matter 8 (1996) 5415.

[9] P. S. Sandu, J. H. Kim, and J. S. Brooks: Phys Rev. B56 11566 (1997).

[10] So-Y. Han, J. H. Kim, and J. S. Brooks: ICSM98 proceedings.

[11] J. Y. Fortin and T. Ziman: Phys. Rev. Lett. 80 (1998) 3117.

[12] M. Nakano: J. Phys. Soc. Jpn. 66 (1997) 19.

[13] A. S. Alexandrov and A. M. Bratkovsky: Phys. Lett. A 234 (1997) 53.

[14] Quasi-two-dimensional organic conductors $(\kappa \text {-(BEDT-TTF })_{2} \mathrm{Cu}(\mathrm{NCS})_{2}$ and $\left.\alpha-(\mathrm{BEDT}-\mathrm{TTF})_{2} \mathrm{KHg}(\mathrm{SCN})_{4}\right)$ have the two energy bands as shown in Fig. 1(a). In quasi-two-dimensional material, $\mathrm{Sr}_{2} \mathrm{RuO}_{4}$, three closed orbits $(\alpha, \beta$ and $\gamma)$ exist. The contribution of the magnetic breakdown for the Fermi surface of $\mathrm{Sr}_{2} \mathrm{RuO}_{4}$ is much smaller than that of the quasi-twodimensional organic conductors.

[15] F. A. Meyer, E. Steep, W. Biberacher, P. Christ, A. Lerf, A. G. M. Jansen, W. Joss, P. Wyder and K. Andres: Europhys. Lett. 32 (1995) 681.

[16] S. Uji, M. Chaparala, S. Hill, P. S. Sandhu, J. Qualls, L. Seger and J. S. Brooks: Synth. Met. 85, (1997) 1573.

[17] M. M. Honold, N. Harrison, M. S. Nam, J. Singleton, C. H. Mielke, M. Kurmoo, and P. Day: Phys. Rev. B58 (1998) 7560.

[18] R. Settai, Y. Yoshida, A. Mukai and Y. Onuki: unpublished

[19] K. Yamaji: J. Phys. Soc. Jpn. 58 (1989) 1520.

[20] M. Nakano: J. Phys. Soc. Jpn. 68 (1999) 1801.

[21] E. Ohmichi, Y. Maeno and T. Ishiguro: J. Phys. Soc. Jpn. 68 (1999) 24

[22] H. Shiba and H. Fukuyama: J. Phys. Soc. Jpn. 26 (1969) 910.

[23] R. W. Stark and R. Reifenberger: J. Low Tem. Phys. 26 (1977) 763.

[24] Y. Yoshida, R. Settai, Y. Onuki, H. Takei, K. Betsuyaku and H. Harima: J. Phys. Soc. Jpn. 67 (1998) 1677. 